\title{
Nonlinear Model Predictive Control of a Robotic Soft Esophagus
}

This paper was downloaded from TechRxiv (https://www.techrxiv.org).

\section{LICENSE}

CC BY 4.0

SUBMISSION DATE / POSTED DATE

06-02-2021 / 10-02-2021

\section{CITATION}

Bhattacharya, Dipankar; Hashem, Ryman; Cheng, Leo; Xu, Peter (2021): Nonlinear Model Predictive Control of a Robotic Soft Esophagus. TechRxiv. Preprint. https://doi.org/10.36227/techrxiv.13725673.v1

DOI

10.36227/techrxiv.13725673.v1 


\title{
Nonlinear Model Predictive Control of a Robotic Soft Esophagus
}

\author{
Dipankar Bhattacharya, Ryman Hashem, Leo Cheng, \\ and Weiliang $\mathrm{Xu}$, Senior Member, IEEE
}

\begin{abstract}
Strictures caused by esophageal cancer can narrow down the esophageal lumen, leading to dysphagia. Palliation of dysphagia has driven the development of a Robotic Soft Esophagus (RoSE) to provide a novel in vitro platform for esophageal stent testing and food viscosity studies. In RoSE, peristaltic wave generation and control were done in an open-loop manner since the conduit lacked visibility and embedded sensing capability. Hence, in this work, RoSE version 2.0 (RoSEv2.0) was designed with embedded Time Of Flight (TOF) and pressure sensors to measure conduit displacement and air pressure, respectively, for modeling and control. Model Predictive Control (MPC) of RoSEv2.0 was implemented to govern the peristalsis and air pressure profile autonomously. The implemented MPC used Sparse Identification Nonlinear Dynamics with Control (SINDYC) models to estimate the future states of ROSEv2.0. The dynamic models were discovered from the TOF and pressure sensors captured data. Peristalsis waves of speed $20 \mathrm{~mm} / \mathrm{s}$, wavelength $75 \mathrm{~mm}$, and amplitudes $5,7.5$, and $10 \mathrm{~mm}$ were successfully generated by the MPC. Additionally, RoSEv2.0 with the MPC was employed to perform stent migration testing with various food boluses consistencies.
\end{abstract}

Index Terms-Machine Learning, Model Predictive Control, Predictive Models, Robotic Soft Esophagus, Soft robotics, Time of Flight Distance Sensor

\section{INTRODUCTION}

Esophageal cancer can cause esophageal stricture, which distresses lumen patency, leading to dysphagia [1]. Esophageal strictures can be addressed by implanting an endoprosthetic stent in the esophagus, which can hold open the esophagus and provide relief to patients [2]. However, stent migration caused by the stent interaction with the continuous peristaltic waves, is a significant shortcoming of concern to the patients [3]. Hence, a Robotic Soft Esophagus (RoSE) has been developed as an alternative platform for conducting stent testing under bolus swallow conditions [4].

Manuscript received Month $\mathrm{xx}, 2 \mathrm{xxx}$; revised Month $\mathrm{xx}, \mathrm{xxxx}$; accepted Month $x, x x x x$. This work was supported financially by Riddet Institute, Palmerston North, New Zealand, which is a national center of research excellence. (Corresponding author: Weiliang $\mathrm{Xu}$.)

D. Bhattacharya, R. Hashem and W. Xu are with the Department of Mechanical Engineering, University of Auckland, Auckland 1010, New Zealand (e-mail:,dbha483@aucklanduni.ac.nz; ryman_87@live.com; p.xu@auckland.ac.nz).

L. K. Cheng is with Auckland Bioengineering Institute, University of Auckland, Auckland 1010, New Zealand (e-mail:, I.cheng@ auckland.ac.nz).
Model Predictive Control (MPC) is an advanced and wellknown technique of controlling highly nonlinear processes with constraints [5], [6]. Based on the past observations, MPC employs the concept of receding horizon (forward shifting of prediction horizon) to anticipate potential disturbances and thereby generating a control sequence that is a proposition among possible alternatives that can manage disturbances well at the next time step [5], [7]. Unlike traditional control theory, MPC is effective if there is a process dead-time or if the setpoint/reference trajectory is well defined ahead of time. Since the 1980s, there is a dramatic increase in the application of MPC in industries [7] like oil refineries [8], power systems [9], and robotics [10], because MPC can model and thereby predict nonlinear and non-minimum phase dynamics systems very accurately [11].

Advanced control techniques like the MPC relies on developing a suitable plant model for optimum performance. Soft robots like the RoSE [12] and soft robotic gastric simulator [13] manufactured by soft elastomers like silicone rubber, cannot be modeled from the perspective of first principles due to their complex, continuous, and highly compliant intrinsic deformation, which leads to infinite degrees of freedom [14]. Developments in the area of Machine Learning (ML) and data science have opened the gateway to model RoSE with data-driven techniques [15]. The Sparse identification of Nonlinear Dynamics with Control (SINDYC) approach [16], [17], has succeeded in addressing challenges in the data-driven modeling of RoSE [15]. Bhattacharya et al. has proven that the SINDYC algorithm promotes sparsity by identifying a few linear and nonlinear terms governing the dynamics of the RoSE [15]. Additionally, unlike other ML techniques like the Artificial Neural Networks (ANN), SINDYC demands less training data, less execution time, and avoids overfitting [18]. Integrating the RoSE SINDYC model in the MPC framework can take advantage of the underlying dynamics in achieving the prescribed peristalsis profile for stent testing.

The primary aim of this paper is to actuate the RoSE conduit with pre-defined peristaltic wave trajectories by implementing SINDYC-MPC framework. The fulfillment of the aim will make RoSE capable of generating various peristaltic wave shapes, which will further enhance the in vitro stent migration testing potential of RoSE. Five significant steps were followed to implement the MPC for stent migration testing. First, Robotic Soft Esophagus version 2.0 (RoSEv2.0) was developed with an embedded array of Time of Flight (TOF) sensors to address the issue of limited visibility inside 
the RoSEv2.0 conduit. Second, RoSEv2.0 was actuated with various pressure varying trajectories and data from TOFs and Valve Pressure Sensors (VPSs) were collected. Third, discretetime formulation of SINDYC referred to as Discrete Time SINDYC (DTSINDYC) was applied to the collected data to discover Discrete Time Differential Equation (DTDE) models of RoSEv2.0. Fourth, nonlinear MPC using the DTDE models were designed and implemented to control the peristaltic motion of the RoSEv2.0 conduit. Last, stent migration data for a candidate stent were recorded for various peristalsis speed and bolus swallow conditions.

The research makes several noteworthy contributions to the field of soft robotics. This research has solved the sensing issue for modeling and control of RoSE by introducing RoSEv2.0 (improved version of RoSE) with TOF sensors. Additionally, to the best of our knowledge, the SINDYC-MPC framework presented in this research has been applied for the first time in a soft robot. The closed-loop control in the form of MPC has been implemented for the first time in RoSEv2.0. In this research, the presented modeling and control methodology is not a robot or sensor specific, and it can be extended to any soft-robotic system with any sensor provided input-output datasets can be generated. Hence to verify the versatility of this approach, MPC of RoSEv2.0 controling the chamber air pressure was implemented with VPSs by following the same implementation methodology described with the TOF sensors.

\section{Problem Formulation}

The foundation of supervised $\mathrm{ML}$ techniques like the SINDYC is qualitative and quantitative data collection. To a greater extent, the quality of the training data determines the SINDYC modeling generalization when applied to any soft robot like RoSE [12]. The updated RoSE (RoSEv2.0) has 12 identical layers ( $L_{1}$ to $L_{12}$ ) such that each layer contains four air pressure chambers surrounding the conduit axis symmetrically. Each horizontal whorl of four chambers at each layer is connected to an electro-pneumatic pressure valve (ITV-0030-3BS, SMC, USA) such that RoSEv2.0 has 12 independent inputs to control the actuation of RoSEv2.0. In contrast with RoSE, RoSEv2.0 is developed with embedded sensing capability. The significant variation between RoSE and RoSEv2.0 is the outer casing construction, which is replaced with a transparent Liquid Polydimethylsiloxane (PDMS) (SYLGARD 182, Dow, USA) casing in RoSEv2.0 (Fig. 1). An array of TOF (VL6180X, STMicroelectronics, Switzerland) sensors are placed on top of the PDMS layer to measure the conduit deformation laterally from the outside.

The DTSINDYC models $M_{1}$ and $M_{2}$ based on TOF and VPS, respectively (Table I) for implementing the MPC are built on a small number of datasets collected from the RoSEv2.0 sensors and aims to discover the underlying DTDEs given by (1) such that the equations best-fit the captured dataset.

$$
\mathbf{x}_{k+1}=\mathbf{g}\left(\mathbf{x}_{k}, \mathbf{u}_{k}\right)
$$

In (1), $\mathbf{u}_{k} \in \mathbb{R}^{n}$, and $\mathbf{x}_{k} \in \mathbb{R}^{n}$ represent the digital values for pressure command applied to the pneumatic valves

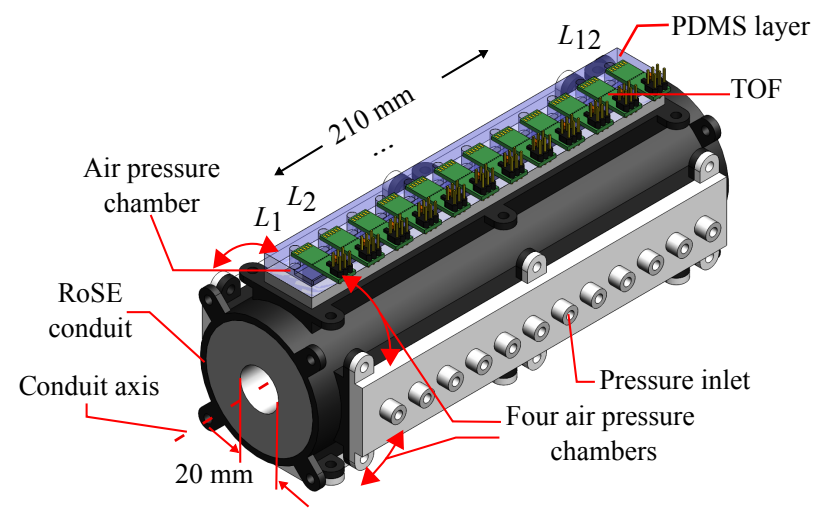

Fig. 1. Isometric view of RoSEv2.0 CAD model.

TABLE I

CHARACTERISTICS OF THE DTSINDYC MODELS.

\begin{tabular}{ccccc}
\hline \hline Model & Sensor used & State variables & Representation & $\begin{array}{c}\text { Control actuation } \\
\text { type }\end{array}$ \\
\hline$M_{1}$ & TOF & $x_{k, 1}, x_{k, 2}, x_{k, 3}$ & Displacement (mm) & Peristaltic \\
$M_{2}$ & VPS & $x_{k, 1}, x_{k, 2}, x_{k, 3}$ & Pressure $(\mathrm{kPa})$ & Peristaltic \\
\hline \hline
\end{tabular}

at $k^{\text {th }}$ time step to govern the RoSEv2.0 actuation and its corresponding TOF or VPS measurements, respectively. $\mathbf{g}($. represents a nonlinear polynomial function consisting of first and second order polynomial, and constant terms, mapping $\mathbb{R}^{n} \times \mathbb{R}^{n} \rightarrow \mathbb{R}^{n}$. Equation 1 also represents the predictive nature of the DTSINDYC models of RoSEv2.0, which will be used for implementing the MPC. At any time-instant $k$, given $\mathbf{x}_{k}$ and $\mathbf{u}_{k}$ of RoSEv2.0, the future of the RoSEv2.0 states denoted by $\mathbf{x}_{k+1}$ can be predicted. In this research, $\mathrm{g}$ (.) represents two different DTSINDYC models $M_{1}$ and $M_{2}$, generated from the VPS and TOF captured data, respectively.

Table I provides the description and physical representation of the state variables associated with the models. The models are used in the MPC for controlling conduit displacement or chamber air pressure in case of peristaltic actuation [4]. Since at least three RoSEv2.0 layers are required to match the human esophagus peristalsis wavelengths [19], thus by emphasizing the TOF sensors measurement accuracy, layer $L_{5}, L_{6}$, and $L_{7}$ were chosen. The MPC of RoSEv2.0 using $M_{1}$, or $M_{2}$ is implemented to compute the control law $\mathbf{u}\left(. \mid \mathbf{x}_{k}\right)=\left\{\mathbf{u}_{j+1}, \cdots, \mathbf{u}_{j+k}, \cdots, \mathbf{u}_{j+N_{u}}\right\}$ at any time-step $j$, for achieving a prescribed peristaltic actuation profile of the robot's conduit over a control horizon $N_{u}$ and prediction horizon $N_{p}$, provided the current TOF or VPS measurement $\mathbf{x}_{j}$ is available. Hence, the implicit feedback law for controlling the valves and hence, controlling the RoSEv2.0 can be written as

$$
\mathbf{u}\left(j+1 \mid \mathbf{x}_{j}\right)=\mathbf{u}_{j+1}
$$

where $\mathbf{u}_{j+1}$ is the first row in the optimized actuation sequence, representing pressure commands applied to the $L_{5}$, $L_{6}$ and $L_{7}$ pneumatic valves, beginning at the initial condition $\mathbf{x}_{j}$. 


\section{RoSEv2.0 FABRICATION, ACtUATION, SENSING, AND STATE DEFINITION}

RoSEv2.0 is built utilizing custom-designed mold and housings. Room-Temperature-Vulcanizing (RTV) silicone rubber material (Ecoflex 0030, Smooth-on, USA) in its uncured state is poured into the housing, rod, and chamber-placeholder assembly and left seated for vulcanization at room temperature (Fig. 2 (a)). After hardening of the silicone rubber, the chamber placeholders are removed (Fig. 2 (b)). Simultaneously, the PDMS (SYLGARD 182, Dow, USA) outer layer is also cast and removed from its molds in a similar manner (Fig. 2 (c) and (d)). Subsequently, side $S_{1}$ of RoSEv2.0 and the PDMS layer are bonded together with an adhesive (Sil-Poxy, Smooth-On, USA) (Fig. 2 (e)). With the same adhesive on $S_{1}$, an array of TOF sensors is attached on top of the PDMS layer (Fig. 2 (f)). Outer covers, pneumatic tubes, and tees are attached to RoSEv2.0 to assure that the silicone rubber deformation occurs inwards (Fig. 2 (g)).

The entire RoSEv2.0 conduit $L_{1}$ to $L_{12}$ does not contain any rigid skeletal boundary, and when pressurized with air, the whorl of four chambers per layer inflate and occlude, mimicking circular muscle activation. The pneumatic valves corresponding to each RoSEv2.0 layer are interfaced with a Raspberry Pi 4 Model B through an Analog to Digital Converter (ADC) and Digital to Analog Converter (DAC) board. Custom firmware protocols for generating peristalsis, written in Python 3.7, are developed on the Pi to actuate the robot in open-loop, with continuous, independent variable pressure trajectories as discussed in section II. By utilizing the peristaltic protocols, training and validation data for the DTSINDYC models are collected to derive and test $M_{1}$ and $M_{2}$.

The TOFs are initially calibrated for range-offset and crosstalk to operate through the PDMS. The TOFs are further calibrated with a webcam (C922, Logitech, Switzerland) by implementing Python OpenCV CSRT tracker algorithm (Fig. 2 (g) and (h)). For webcam calibration, two hemispherical $4 \mathrm{~mm}$ retroreflective markers are placed, one for tracking and another for reference. Both TOF and webcam measurements are taken simultaneously by inflating the RoSEv2.0 chambers with the peristaltic actuation protocol. The time-series measurements are compared to determine additional offsets and scaling factors.

The measured RoSEv2.0 conduit displacement data at the center of $L_{5}, L_{6}$, and $L_{7}$ air pressure chamber of side $S_{1}$ are considered as the states of RoSEv2.0. Hence, three discrete states $x_{k, 1}, x_{k, 2}$, and $x_{k, 3}$ corresponding to layer $L_{5}, L_{6}$, and $L_{7}$ are defined as RoSEv2.0 states (Fig. 2 (i)). Each state represents $x$-axis conduit displacement at $L_{5}, L_{6}$, and $L_{7}$ of $S_{1}$, respectively. For model simplification, it is assumed that the conduit thickness $t$ remains unchanged throughout its deformation. Additionally, it is also considered that the deformation of the adjacent chambers in each layer is symmetric, and displacement along $y$ and $z$-axis are negligible [15].

\section{DISCRETE TIME SINDYC}

The original SINDYC algorithm can be extended to discrete-time dynamical systems, represented by (1) [20].
There are two primary reasons for implementing the DTSINDYC over the conventional one. Firstly, the calculation of a derivative from noisy data is not required for the DTSINDYC algorithm, which is a significant advantage over its continuous-time counterpart. Secondly, a discretized model of RoSEv2.0 is necessary for the implementation of MPC.

The data collected from the VPSs or the TOF $\left(x_{1, k}\right.$, $x_{2, k}$, and $x_{3, k}$, (Fig. 2 (i)) sensors are arranged into two $m \times n$ matrices $\mathbf{X}_{1}^{m}=\left[\mathbf{x}_{1}^{T}, \mathbf{x}_{2}^{T}, \cdots, \mathbf{x}_{m}^{T}\right]^{T}$ and $\mathbf{X}_{2}^{m+1}=$ $\left[\mathbf{x}_{2}^{T}, \mathbf{x}_{3}^{T}, \cdots, \mathbf{x}_{m+1}^{T}\right]^{T}$. Likewise, the input data are stored in an $m \times l$ matrix $\mathbf{U}=\left[\mathbf{u}_{1}^{T}, \mathbf{u}_{1}^{T}, \cdots, \mathbf{u}_{m}^{T}\right]^{T}$. Variables $m$, $n$, and $l$ represents the number of samples, state variables $\left(x_{k, 1}, x_{k, 2}\right.$, and $x_{k, 3}, n=3$ ), and inputs, respectively and $\mathbf{x}_{q}=\left[x_{q, 1}, x_{2, q}, \cdots, x_{q, n}\right]^{T} \mid q \in\{1, \cdots, m+1\}$.

In this research, the number of inputs is considered equal to the number of outputs $(l=n)$, where inputs and outputs are digital pressure commands to the valves and TOF or VPS measurements, respectively. The DTSINDYC algorithm implements a library of candidate functions matrix $\Theta$ of order $m \times p$, consisting of constant and polynomial (of different order) terms. The library matrix $\Theta$ can be defined as:

$$
\boldsymbol{\Theta}\left(\mathbf{X}_{1}^{m}, \mathbf{U}\right)=\left[\begin{array}{llllll}
1 & \mathbf{X}_{1}^{m} & \mathbf{U} & \mathbf{X}_{1}^{m} \otimes \mathbf{X}_{1}^{m} & \mathbf{X}_{1}^{m} \otimes \mathbf{U} & \ldots
\end{array}\right]
$$

where, $\mathbf{X}_{1}^{m} \otimes \mathbf{U}$ defines all the possible product combinations of the components of $\mathbf{X}$ and $\mathbf{U}$. Each column of $\boldsymbol{\Theta}\left(\mathbf{X}_{1}^{m}, \mathbf{U}\right)$ represents the potential candidate for the final right-hand side expression for (1). There is an enormous opportunity for decision in building the library function of nonlinearities. Thus, the system in (1) can be written as:

$$
\mathbf{X}_{2}^{m+1}=\boldsymbol{\Theta}\left(\mathbf{X}_{1}^{m}, \mathbf{U}\right) \mathbf{\Upsilon}
$$

where $\boldsymbol{\Upsilon}=\left[\boldsymbol{v}_{0}, \boldsymbol{v}_{1}, \cdots, \boldsymbol{v}_{n-1}\right]$ is a sparse matrix of coefficients of order $p \times n$ such that the sparse vectors $\left\{\boldsymbol{v}_{i}\right\}_{i=0}^{n-1}$, lie in the subspace of $\mathbb{R}^{p}$. The sparsity of $\boldsymbol{v}_{i}$ selects the active terms from $\mathbf{\Theta}\left(\mathbf{X}_{1}^{m}, \mathbf{U}\right)$ to identify the $g_{i}$ of $x_{k+1, i}=g_{i}\left(\mathbf{x}_{k}, \mathbf{u}_{k}\right)$ in (1). Since only a few of the candidates will appear in the row of $\mathbf{g}$ in (1), a sparse regression problem can be defined and $\Upsilon$ can be determined by optimizing it [16]. After the determining $\Upsilon$, DTSINDYC model of RoSEv2.0 can be written as:

$$
\mathbf{x}_{k+1}=\mathbf{g}\left(\mathbf{x}_{k}, \mathbf{u}_{k}\right)=\mathbf{\Upsilon}^{T} \boldsymbol{\Theta}\left(\mathbf{x}_{k}^{T}, \mathbf{u}_{k}^{T}\right)^{T}
$$

The DTSINDYC model in (5) reveals the underlying dynamics of RoSEv2.0 in the form of DTDEs.

\section{Overview of Model Predictive Control in RoSEv2.0}

The MPC optimization is perfomed over a receding prediction horizon by forward shifting the prediction horizon at every time step $N_{p}$. At $j^{\text {th }}$ time instant, the MPC applies a reference signal $\mathbf{x}_{j}^{(r e f)}$ to the Cost Function Minimization (CFM) block (Fig. 3). The CFM block minimizes a cost function $J$ over $N_{p}$. The output of the CFM is either used as an input to the valves or DTSINDYC model $\left(M_{1}\right.$ or $M_{2}$, Table I). Since the model in MPC is used for prediction; 


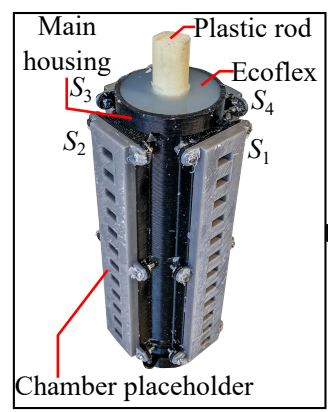

(a)

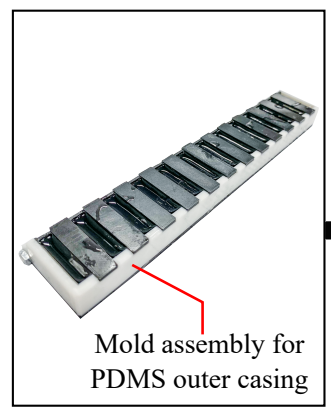

(c)

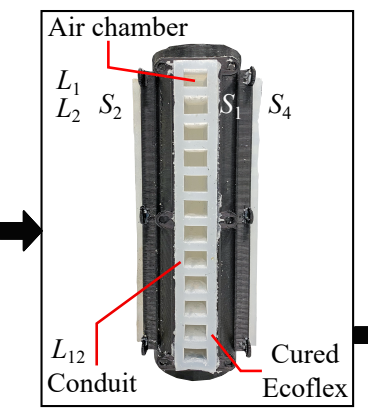

(b)

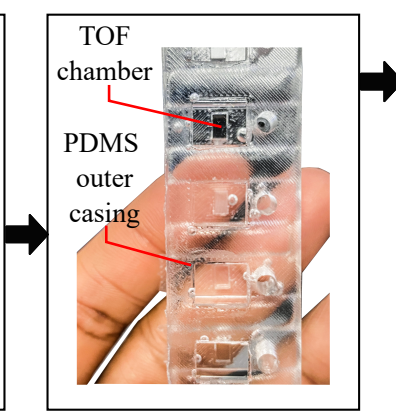

(d)

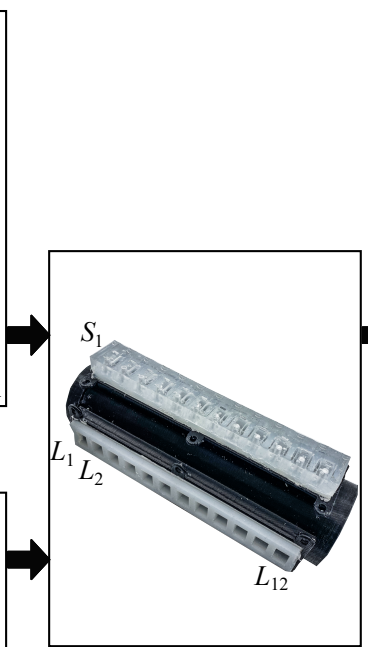

(e)

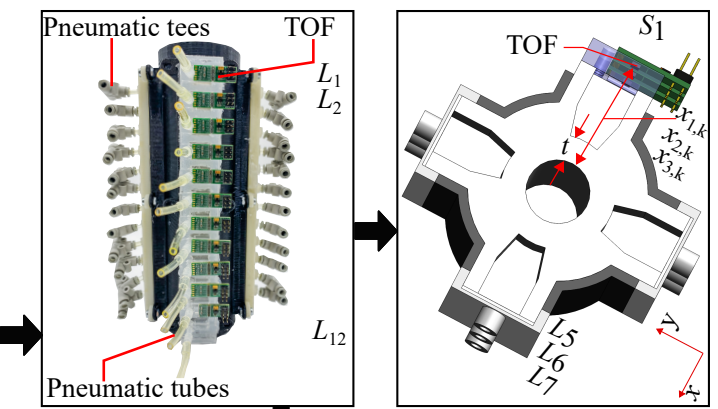

(i)

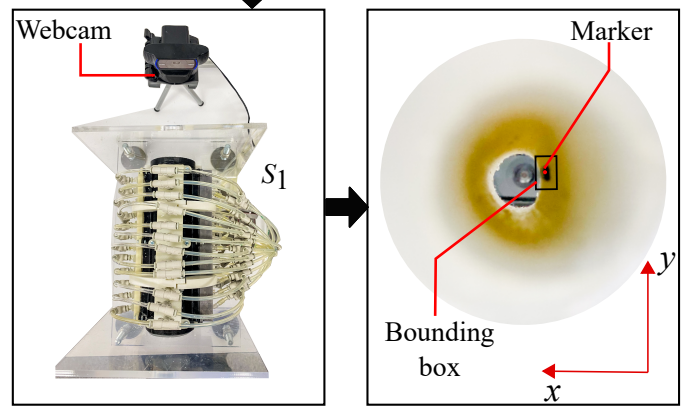

(g)

(h)

Fig. 2. (a) to (f) Construction of RoSEv2.0. (g) to (h) Webcam setup for TOF calibration. (i) RoSEv2.0 sectional view for defining the states.

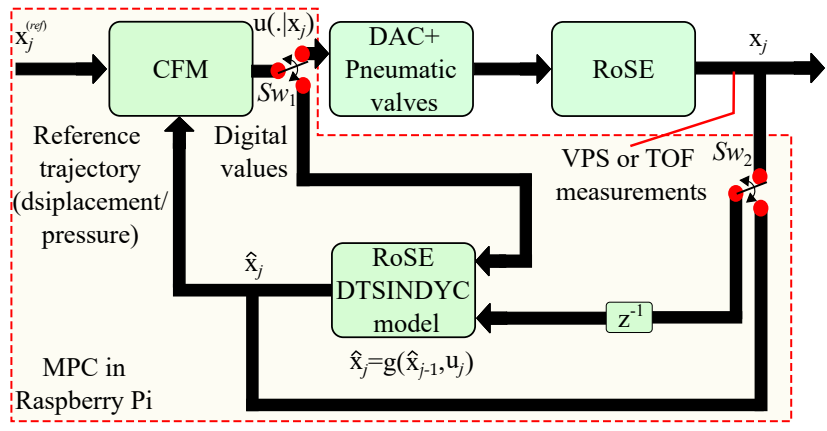

Fig. 3. Block diagram of DTSINDYC-MPC explaining its functional description.

thus, the model output at any instant $j$ is denoted by $\hat{\mathbf{x}}_{j}$. Between $j$ and $j+1$ time step, the Single Pole Double Throw (SPDT) switch $S w_{1}$ is set to the DTSINDYC model whose output is used to calculate a set of optimal control values $\mathbf{u}_{. \mid \mathbf{x}_{j}}:=\left\{\mathbf{u}_{j+1}, \cdots, \mathbf{u}_{j+k}, \cdots, \mathbf{u}_{j+N_{c}}\right\}$ over a control horizon $N_{c}$. The behavior of RoSEv2.0 over $N_{p}$ is estimated by its model. To determine the sequence of estimations $\left\{\hat{\mathbf{x}}_{j}, \hat{\mathbf{x}}_{j+1}, \cdots, \hat{\mathbf{x}}_{j+k}, \cdots, \hat{\mathbf{x}}_{j+N_{p}-1}\right\}, S w_{2}$ is initially set to the RoSEv2.0 TOF or VPS for $\hat{\mathbf{x}}_{j}$ and then to the model output for the subsequent predictions. Next, when the CFM algorithm has solved for the best input, $S_{1}$ is moved to the valves and first control value from $\mathbf{u}_{j+1}$ is applied to the valves. At next time instant $j+1$, the computation is repeated with horizon moved by one time-step. The implicit control law for each time-step is given by (2). The cost function optimized by the CFM block at each time-step can be written as:

$$
\begin{aligned}
& \arg \underset{\mathbf{u}_{\cdot \mid \mathbf{x}_{j}}}{\min } J\left(\mathbf{x}_{j}\right)=\arg \min _{\mathbf{u}_{\cdot \mid \mathbf{x}_{j}}}\left\{\sum_{k=0}^{N_{p}-1}\left\|\hat{\mathbf{x}}_{j+k}-\mathbf{x}_{j+k}^{(r e f)}\right\|_{\mathbf{Q}}^{2}+\right. \\
& \left.\sum_{k=0}^{N_{c}-1}\left(\left\|\mathbf{u}_{j+k}\right\|_{\mathbf{R}_{\mathbf{u}}}^{2}+\left\|\mathbf{u}_{j+k}-\mathbf{u}_{j+k-1}\right\|_{\mathbf{R}_{\Delta \mathbf{u}}}^{2}\right)\right\} \\
& \text { s.t. } \hat{\mathbf{x}}_{j+k}=\mathbf{g}\left(\hat{\mathbf{x}}_{j+k-1}, \mathbf{u}_{j+k-1}\right), \mathbf{u}_{l b} \leq \mathbf{u}_{k} \leq \mathbf{u}_{u b}
\end{aligned}
$$

At every iteration (time-step) $j$, CFM minimizes the $J$ in (6). The leftmost term in (6) penalizes the root mean square deviations of the RoSEv2.0 model predicted trajectory $\hat{\mathbf{x}}_{j+k}$ (given by (7)), from the reference trajectory $\mathbf{x}_{j+k}^{(r e f)}$. Control output sequence $\mathbf{u}_{j+k}$ and change in control output sequence $\left(\mathbf{u}_{j+k}-\mathbf{u}_{j+k-1}\right)$ are also penalized by the middle and the rightmost terms in (6), respectively. The range of $\mathbf{u}_{k}$ at every iteration of MPC is constrained by control bounds $\left(\mathbf{u}_{l b}\right.$ and $\mathbf{u}_{u b}$ in (7)). In (6), the weight matrix $\mathbf{Q}$ is positive semi-definite and matrices $\mathbf{R}_{\mathbf{u}}$ and $\mathbf{R}_{\boldsymbol{\Delta} \mathbf{u}}$ are positive definite.

\section{MPC DEsign And IMPLEMEntation IN RoSEv2.0}

This section ties together section III to $\mathrm{V}$ in order to design and implement the MPC in RoSEv2.0. In the domain of mathematics, the biological peristaltic waves are typically modeled as per the sinusoidal waveform given by (8) [21], [22].

$H(z, t)= \begin{cases}\epsilon+\frac{\alpha}{2}\left[1-\cos \left(2 \pi \frac{z-c t}{w}\right)\right] & , c t \leq z \leq(c t+w) \\ \epsilon & , \text { elsewhere }\end{cases}$ 
In (8), $\epsilon$ is minimum radius $(\mathrm{mm}), z$ is axial displacement $(\mathrm{mm}), t$ is time $(s), H(z, t)$ is conduit's radius as a function of $x$ and $t, \alpha$ is amplitude of the wave $(\mathrm{mm}), c$ is velocity of the travelling wave $\left(\mathrm{mm} . \mathrm{s}^{-1}\right)$, and $w$ wavelength $(\mathrm{mm})$. Equation 8 is a modified version of the orginal equation described by [19], [21] to incorporate the entire ascending half-cycle (full wavelength). By considering $z=z_{d},\left(z_{d}\right.$ is the axial adjacent chamber spacing, which is $15 \mathrm{~mm}), t_{d}=z_{d} / c$ continuous time frequency $f_{d}=c / w$, and $t=k T_{s}$, discretetime form of (8) can be written as

$$
H(k)=\quad \epsilon+\frac{\alpha}{2}\left[1-\cos \left\{\omega\left(k-\frac{t_{d}}{T_{s}}\right)\right\}\right]
$$

where, discrete frequency $\omega=2 \pi f_{d} / F_{s}$, sampling frequency $F_{s}=1 / T_{s}$, and $t_{d} / T_{s} \leq k \leq\left(t_{d} / T_{s}+1 / T_{s} f_{d}\right)$. The peristaltic waveform profile, given by (9) can be accomplished by controlling time-varying pressure trajectories into the adjacent whorl of chambers simultaneously (Fig. 4). The pneumatic valves associated with each layer of RoSEv2.0 command the pressure profile in each layer. The role of the MPC is to find out the optimal control law in terms of digital values, which are supplied to the valves to achieve peristaltic wave shapes, defined by the parameters ( $c$ and $w / 2$ ) of (9).

Next, three time-shifted versions of (9) for layer $L_{5}, L_{6}$, and $L_{7}$ of RoSEv2.0 are formulated $\left(x_{k, n}^{(r e f)}=H_{n}(k) \mid n=1,2,3\right)$ as per (10).

$$
x_{k, n}^{(r e f)}=\epsilon+\frac{\alpha}{2}\left[1-\cos \left\{\omega\left(k-(n-1) \frac{t_{d}}{T_{s}}\right)\right\}\right]
$$

In (10), $(n-1) t_{d} / T_{s} \leq k \leq(n-1)\left(t_{d} / T_{s}+1 / T s f_{d}\right) \mid n=$ $1,2,3$. At a time-instant $j$, the MPC loop receives three present $\left(\left\{x_{j, n}^{(r e f)}=H_{j, n} \mid n=1,2,3\right\},(10)\right)$ and a set of future reference signal values, and feedback value $\left(\left\{x_{j-1, n} \mid n=1,2,3\right\}\right)$ from TOF sensors or VPSs located at layer $L_{5}, L_{6}$ and $L_{7}$ of RoSEv2.0 (Fig. 4). The blocks within the red dashed section of Fig. 3 represents the expanded MPC loop in Fig. 4. By following the minimization procedure discussed in section $\mathrm{V}$, the MPC loop generates three control values $u_{j+1,1}, u_{j+1,2}$ and $u_{j+3,3}$, which are stored in the $L_{2}, L_{3}$ and $L_{4}$ index of the accumulator. $L_{1}$ index is not used since bolus feeder pipes are inserted in RoSEv2.0 conduit and the pipes coincide with $L_{1}$ and $L_{12}$ conduit locations. Hence, to keep the orientation of the feeder pipes unchanged, layers $L_{1}$ and $L_{12}$ are not actuated.

In the next iteration $(j+1)$, a new set of control signals $\left(u_{j+2,1}, u_{j+2,2}\right.$ and $\left.u_{j+2,3}\right)$ are generated and the earlier set of signals are right-shifted by three in the accumulator. It takes $3 q+1$ shifts (iterations) for $u_{j+1,1}, u_{j+1,2}$ and $u_{j+1,3}$ to reach $L_{5}, L_{6}$ and $L_{7}$ respectively, where $q$ is the number of time steps between the peaks of consecutive reference signal given by discrete time delay $q=\left\lfloor t_{d} / T_{s}\right\rfloor$ of (9). If $c=20$ $\mathrm{mm} / \mathrm{s}, x_{d}=15 \mathrm{~mm} w=120 \mathrm{~mm}$ and $T_{s}=0.1 \mathrm{~s}$, then $t_{d}=0.75 \mathrm{~s}, f_{d}=1 / 6 \mathrm{~s}^{-1}$ and $q=7$. Here, $T s$ is the time taken by an MPC loop to complete one cycle, which can vary upon conditions like reference signal type and model complexity, prediction horizon and input bounds. With this kind of approach, the MPC has been made robust to adapt for various peristalsis waveform speeds and wavelengths. The

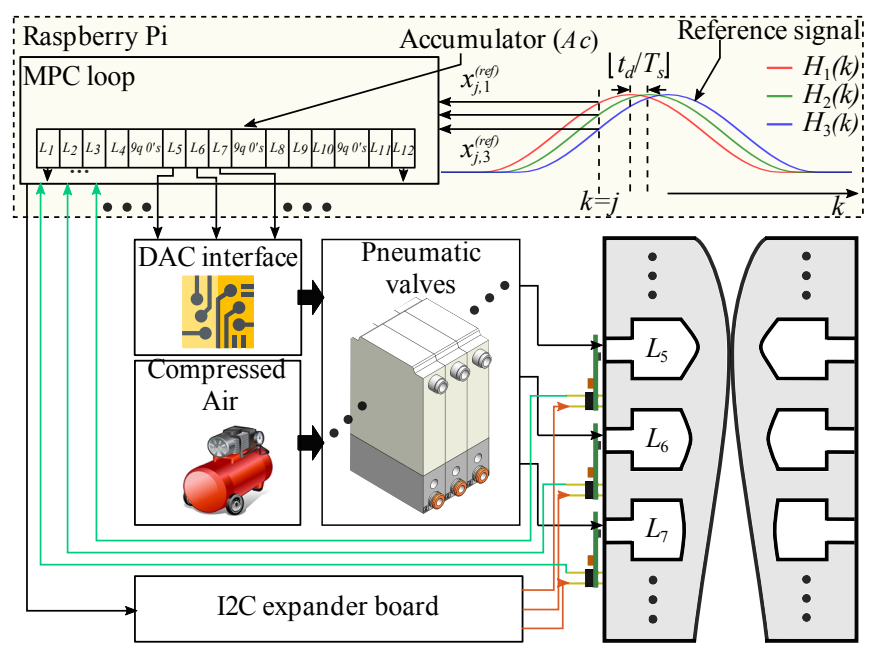

Fig. 4. Block diagram of DTSINDYC-MPC design and implementation in RoSEv2.0.

only downside to this approach is difficulty in achieving higher wave speeds if $T s$ increases. For best and fast optimization, the MPC was tuned for various prediction horizon lengths, weight matrices and control input bounds.

\section{REsults}

\section{A. DTSINDYC Models for Peristaltic Actuation}

This section presents the results with the DTSINDYC modeling methodology presented in section IV. Layer $L_{5}, L_{6}$, and $L_{7}$ of the conduit were actuated with time-shifted staircase waveforms of varying amplitude and time (Fig. 5 (a)) to apply DTSINDYC. It was found that to profile the conduit as per (10) with different waveform speed and diameter; the conduit layers must be actuated with the waveforms as mentioned earlier. The amount of time-shift governs the speed of the peristalsis in RoSEv2.0 and the range of the peristalsis speed was obtained from the RoSE articulography results presented by Dirven et al. [19].

A dataset of size $4350 \times 3$ comprising various amplitude and time-shifted staircase waveform levels (Fig. 5 (a)) and their respective TOF output was collected (orange and blue waveforms in Fig. 5 (b) to (d)). Model $M_{1}$ discovered by applying DTSINDYC [23] to the collected dataset is given below:

$$
\begin{aligned}
{\left[\begin{array}{l}
x_{k+1,1} \\
x_{k+1,2} \\
x_{k+1,3}
\end{array}\right]=} & {\left[\begin{array}{ccc}
0.85 & 0.04 & \left(-0.01-0.02 x_{k, 2}\right) \\
0.02 & 0.84 & \left(-0.17-0.02 x_{k, 2}\right) \\
0 & 0 & 0.91
\end{array}\right]\left[\begin{array}{l}
x_{k, 1} \\
x_{k, 2} \\
x_{k, 3}
\end{array}\right] } \\
+ & {\left[\begin{array}{ccc}
0.05 & 0.02 & -0.01 \\
0.01 & 0.05 & -0.02 \\
0 & 0.02 & +0.02
\end{array}\right]\left[\begin{array}{l}
u_{k, 1} \\
u_{k, 2} \\
u_{k, 3}
\end{array}\right]-\left[\begin{array}{l}
0.57 \\
0.37 \\
0.29
\end{array}\right] }
\end{aligned}
$$

It was found that model $M_{1}$ given by (11), generalize the entire dataset (black dashed-line plots in Fig. 5 (b) to (d)) with a fraction of noisy training data $(m=500$ and $n=3)$, which confirms the robustness of DTSINDYC modeling with low and noisy data availability. 

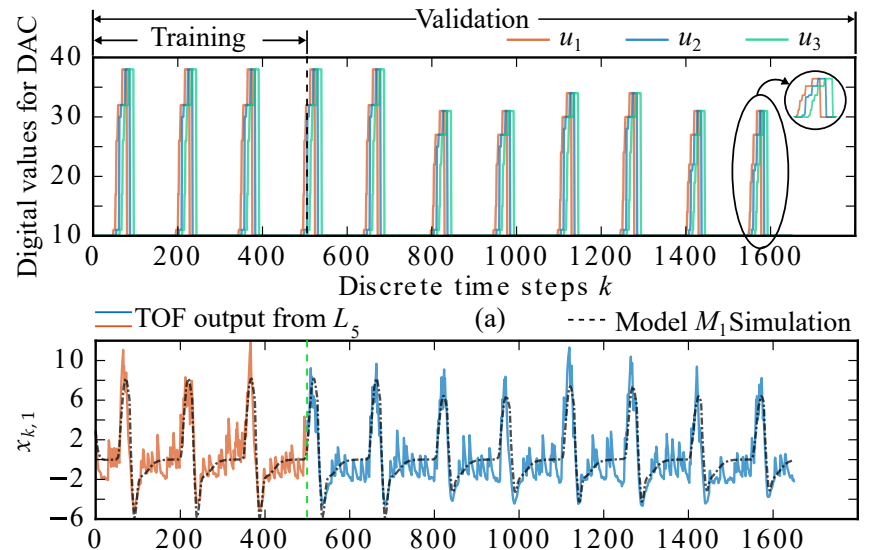

$$
\text { L6L7 }
$$

(b)

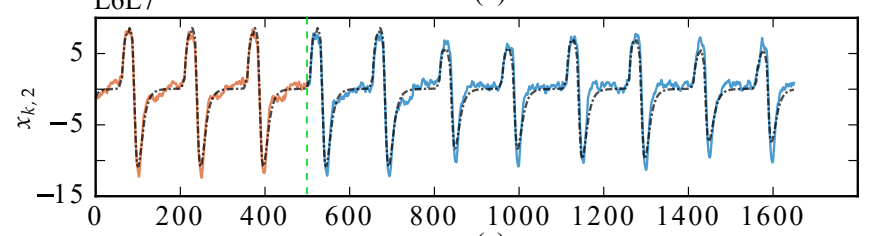

(c)

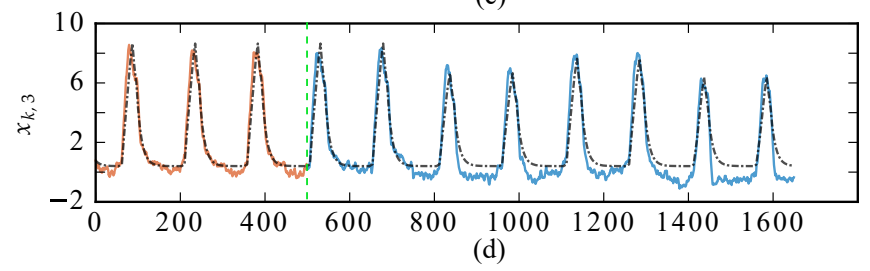

Fig. 5. Plots illustrating the training and validation of model $M_{1}$. (a) The input dataset applied to the $L_{5}, L_{6}$ and $L_{7}$ DACs to generate the output dataset shown by blue and orange plots in (b) to (d). (b) to (d) The dashed black line represents the one-step ahead prediction of $M_{1}$.

Likewise, model $M_{2}$ was derived from the data collected from VPSs located at $L_{5}, L_{6}$, and $L_{7}$. The recorded output from the VPSs exhibited noisy data in the absence of an output filter. Irrespective of the noise, DTSINDYC robustly predicted the state with great accuracy. The algorithm was able to manage the bias-variance trade-off adequately to deliver a precise fit to the data. To emphasize the versatility of DTSINDYC, it is important to note that the procedure followed to built and validate $M_{2}$ was kept same as $M_{1}$, except that the data for the respective models were collected from different sensors.

\section{B. MPC Performance Testing}

The results presented in this section are associated with the theory and methodology presented in section V and VI. The control performance of the implemented MPC with model $M_{1}$ was estimated in terms of Root Mean Square Error (RMSE) $(\mathrm{mm})$ and total execution time (s) taken by the MPC algorithm to stop. Both the performance indicators were evaluated by varying the prediction horizon $N_{p}$ from one to eight, keeping the control horizon $N_{c}=N_{p}$. The evaluation was done prior to the tuning of the control bounds.

To compare the MPC performance of $N_{p}>1$ with $N_{p}=1$ (single-step ahead prediction), the RMSE for $N_{p}=1$ was subtracted from RMSE of all $N_{p}$. From Fig. 6 (a), it can

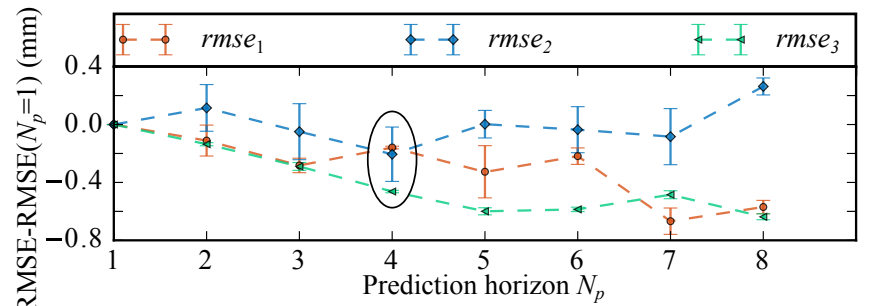

(a)

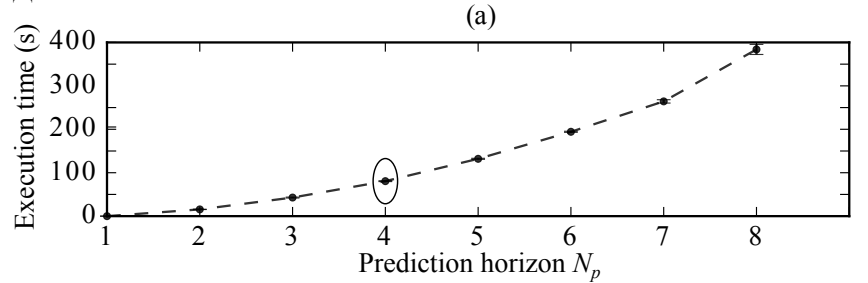

(b)

Fig. 6. Estimation of control performance of $M_{1}$ with different prediction horizon $N_{p}$. (a) RMSE between the RoSEv2.0 controlled output and MPC reference input is plotted with $N_{p}$. (b) Plot showing the total execution time of MPC with the change of $N_{p}$.

be seen that $r m s e_{1}, r^{2 m s e} e_{2}$, and $r m s e_{3}$ decrease up to $N_{p}=4$, where $r m s e_{1}, r m s e_{2}$, and $r m s e_{3}$ are the root mean square error between the reference displacement signals $\left(x_{1}^{(r e f)}, x_{2}^{(r e f)}\right.$, and $\left.x_{3}^{(r e f)}\right)$ and the RoSEv2.0 TOF outputs $\left(x_{1}, x_{2}\right.$, and $\left.x_{3}\right)$ in response to the optimized control law $\left(u_{1}\right.$, $u_{2}$, and $u_{3}$ ). Since the RoSEv 2.0 conduit radius is $10 \mathrm{~mm}$ so a slight increment in the error $(>0.4 \mathrm{~mm})$ will cause a significant impact on the MPC performance. Until $N_{p}=4$ in Fig. 6 (a), all the errors tend to decrease with an increase of $N_{p}$. From Fig. 6 (b), it can be observed that the execution time drastically goes up as $N_{p}$ increments. Since no significant difference in the MPC performance was found for $N_{p}>4$, thus $N_{c}=N_{p}=4$ was chosen.

After selecting a suitable prediction horizon and tuning the control bounds, the performance of MPC with model $M_{1}$ was tested. Reference time-series peristalsis waves $\left(x_{1}^{(r e f)}, x_{2}^{(r e f)}\right.$, and $x_{3}^{(r e f)}$ ) of speed $20 \mathrm{~mm} . \mathrm{s}^{-1}$, wavelength $75 \mathrm{~mm}$, and amplitude 5, 7.5, and $10 \mathrm{~mm}$ (solid line orange plot, Fig. 7 (a) to (c)) were applied to the MPC with an average MPC loop iteration time of $T_{s}=0.15 \mathrm{~s}$.

In Fig. 7 (a) to (c), it can be seen that the three RoSEv2.0 states $\left(x_{1}, x_{2}\right.$, and $x_{3}$ corresponding to $L_{5}, L_{6}$, and $\left.L_{7}\right)$ tracked the prescribed peristalsis trajectory satisfactorily when actuated with the MPC generated control signals $\left(u_{1}, u_{2}\right.$, and $u_{3}$ of Fig. 7 (d)) for the pneumatic valves. It can also be observed that the accuracy of the tracking is far better while inflating the chambers than deflating, which is not a matter of concern because the inflation of the chambers provides shape to the bolus tail and pushes it forward. Hence, half reference wavelength was achieved successfully. Similarly, the implemented MPC with $M_{2}$ and feedback from the VPSs have shown successful tracking of the reference pressure signals (Fig. 8). In Fig. 8, only state $x_{1}$ has been shown. States $x_{2}$, and $x_{3}$ have shown a similar satisfactorily tracking profile. 


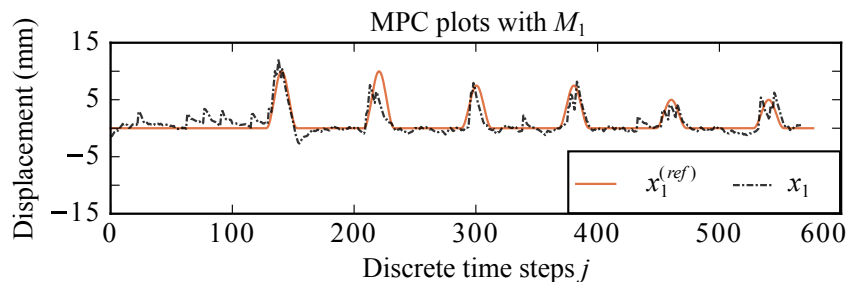

(a)

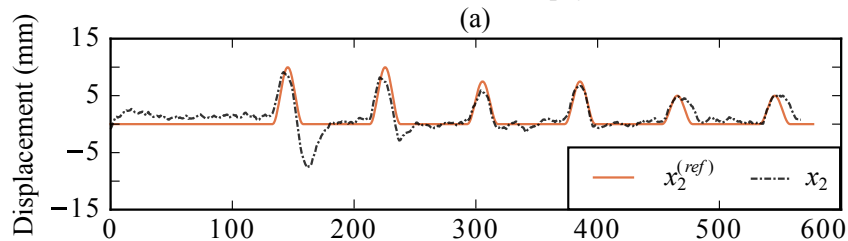

(b)

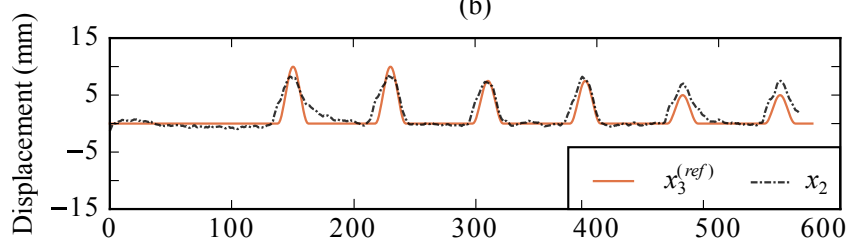

(c)

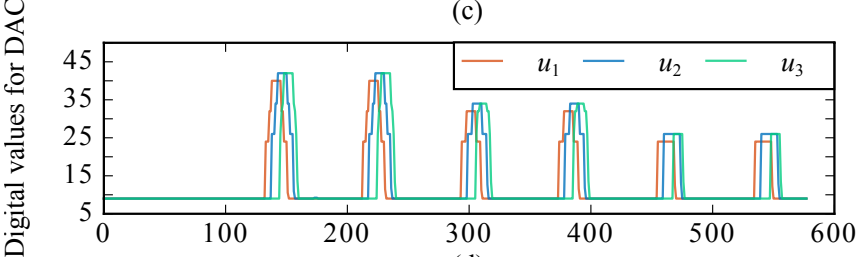

(d)

Fig. 7. MPC performance results with model $M_{1}$. The orange plots in (a) to (c) represent reference sinusoidal waveform trajectories of various amplitudes, applied to the MPC for RoSEv2.0 $L_{5}, L_{6}$, and $L_{7}$. The dashed black line represents the response of the TOFs concerning the control applied by the MPC, shown in (d).

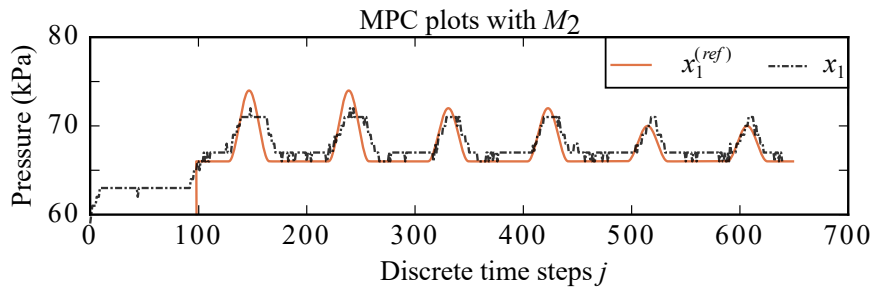

(a)

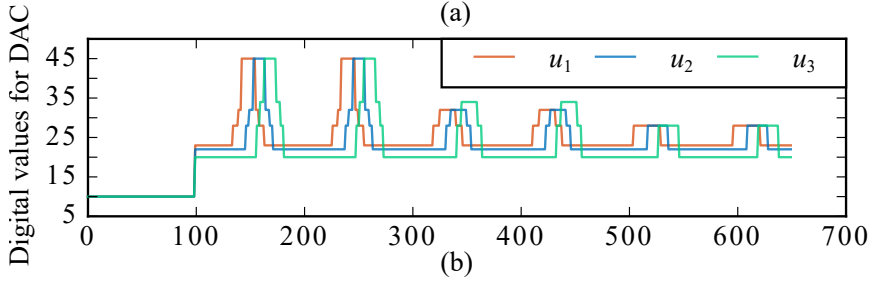

Fig. 8. MPC performance results with model $M_{2}$.

\section{Application of MPC in stent migration testing}

A starch-based fluid thickener (Nutulis, Nutricia, Schiphol, $\mathrm{NL}$ ) was used to replicate the masticated boluses throughout the experimentation [24]. Boluses were formulated by mixing 1,2 , and 3 scoops with $200 \mathrm{ml}$ of water to cover syrup, custard, and pudding-like consistencies, respectively. The associated concentrations and viscosities are 60,80 and $100 \mathrm{~g} / \mathrm{L}$ and $0.45 \pm 0.2,1.2 \pm 0.4$ and $3.0 \pm 1.0 \mathrm{~Pa} / \mathrm{s}$, respectively [19].

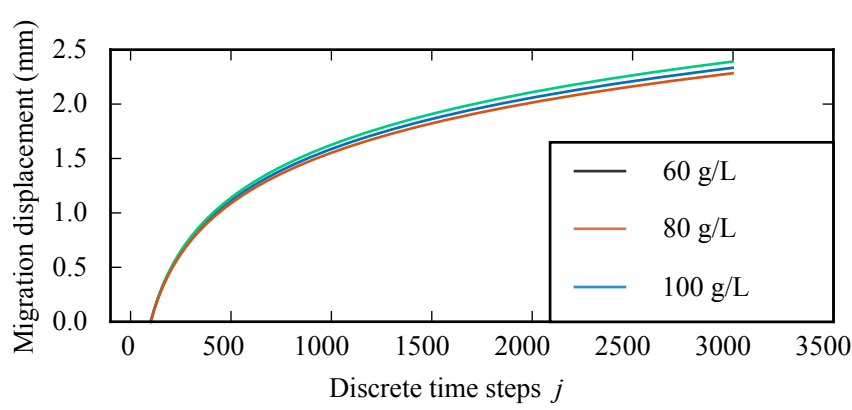

Fig. 9. Stent migration results with the change in bolus concentration conducted for 50 peristalsis cycles.

After the RoSEv2.0 conduit was lubricated with artificial saliva (Aquae Dry Mouth Spray, Hamilton), the candidate stent was deployed in the RoSEv2.0 conduit by using an intruder sheath and a retrieval thread. The complete stent deployment setup is discussed in [4].

Stent migration experiments with bolus swallow were performed on RoSEv2.0 by using peristaltic actuation protocol with 50 peristalsis cycles. The applied methodology for conducting the experiments has been discussed in [4]. From Fig. 9, it can be observed that with the increase in bolus concentration, the migration slightly increases. The obtained results are in line with the results presented in [4] in which the experiments were conducted in an open-loop manner, and the actual conduit displacement was not tracked. Since stent migration depends on the peristalsis parameters, no significant difference in migration results was found compared to the results presented in [4]. But, with the addition of MPC, it can now be assured that prescribed peristalsis has been achieved because in stent implanted RoSEv2.0 experiments, the conduit displacement was controlled by the MPC to ensure occlusion. Hence, implementing MPC has further enhanced the application of stent migration testing in RoSEv2.0.

\section{CONCLUSION}

RoSEv2.0 was developed with an embedded TOF sensors to measure its conduit deformation, which has extended its capabilities. Additionally, with the aid of the integrated TOF sensors, the study has first attempted to implement a closedloop controller in the form of MPC in RoSEv2.0 (Fig. 4). Since, the methodology presented to design the MPC can be extended to any other soft robotic application provided the SINDYC model can be generated, thus MPC was also implemented to control RoSEv2.0 air chamber pressure. The procedure followed for the implementation remained the same, except for the DTSINDYC models, which were identified on VPS data. Additionally, the MPC can now govern the peristalsis profile in RoSE, which is a much-needed requirement for stent testing. Peristalsis waves of speed $20 \mathrm{~mm} / \mathrm{s}$, wavelength $75 \mathrm{~mm}$, and amplitudes $5,7.5$, and $10 \mathrm{~mm}$ were successfully generated by the MPC (Fig. 7). Stent migration testing with different food bolus consistencies were performed 
on RoSEv2.0 to prove that the capability of RoSEv2.0 has been enhanced, after the implementation of the MPC.

\section{REFERENCES}

[1] J. M. Garcia, E. Chambers IV, M. Clark, J. Helverson, and Z. Matta, "Quality of care issues for dysphagia: modifications involving oral fluids," Journal of Clinical Nursing, vol. 19, DOI 10.1111/j.13652702.2009.03009.x, no. 11-12, pp. 1618-1624, 2010.

[2] T. Hanawa, "Materials for metallic stents," Journal of Artificial Organs, vol. 12, DOI 10.1007/s10047-008-0456-x, no. 2, pp. 73-79, 2009.

[3] P. Sharma, R. Kozarek, P. P. C. of the American College of Gastroenterology et al., "Role of esophageal stents in benign and malignant diseases," American Journal of Gastroenterology, vol. 105, DOI 10.1038/ajg.2009.684, no. 2, pp. 258-273, 2010.

[4] D. Bhattacharya, S. J.V. Ali, L. K. Cheng, and W. Xu, "RoSE: A robotic soft esophagus for endoprosthetic stent testing," Soft Robotics, DOI 10.1089/soro.2019.0205.

[5] D. Q. Mayne, J. B. Rawlings, C. V. Rao, and P. O. Scokaert, "Constrained model predictive control: Stability and optimality," Automatica, vol. 36, DOI 10.1016/S0005-1098(99)00214-9, no. 6, pp. 789-814, 2000.

[6] D. W. Clarke, C. Mohtadi, and P. Tuffs, "Generalized predictive controlpart i. The basic algorithm," Automatica, vol. 23, DOI 10.1016/00051098(87)90087-2, no. 2, pp. 137-148, 1987.

[7] C. E. Garcia, D. M. Prett, and M. Morari, "Model predictive control: theory and practice-a survey," Automatica, vol. 25, DOI 10.1016/00051098(89)90002-2, no. 3, pp. 335-348, 1989.

[8] M. Morari and J. H. Lee, "Model predictive control: past, present and future," Computers \& Chemical Engineering, vol. 23, DOI 10.1016/S00981354(98)00301-9, no. 4-5, pp. 667-682, 1999.

[9] J. Golbert and D. R. Lewin, "Model-based control of fuel cells::(1) regulatory control," Journal of power sources, vol. 135, DOI 10.1016/j.jpowsour.2004.04.008, no. 1-2, pp. 135-151, 2004.

[10] X. Hou, S. Guo, L. Shi, H. Xing, Z. Li, D. Xia, M. Zhou, and Y. Liu, "Underwater obstacle avoiding trajectory tracking approach for amphibious spherical robots," in 2020 IEEE International Conference on Mechatronics and Automation (ICMA), DOI 10.1109/ICMA49215.2020.9233669, pp. 1348-1353, 2020.

[11] S. J. Qin and T. A. Badgwell, "A survey of industrial model predictive control technology," Control engineering practice, vol. 11, DOI 10.1016/S0967-0661(02)00186-7, no. 7, pp. 733-764, 2003.

[12] S. Dirven, F. Chen, W. Xu, J. E. Bronlund, J. Allen, and L. K. Cheng, "Design and characterization of a peristaltic actuator inspired by esophageal swallowing," IEEE/ASME Transactions on Mechatronics, vol. 19, DOI 10.1109/TMECH.2013.2276406, no. 4, pp. 1234-1242, 2014.

[13] Y. Dang, Y. Liu, R. Hashem, D. Bhattacharya, J. Allen, M. Stommel, L. K. Cheng, and W. Xu, "Sogut: A soft robotic gastric simulator," Soft Robotics.

[14] D. Rus and M. T. Tolley, "Design, fabrication and control of soft robots," Nature, vol. 521, DOI 10.1038/nature14543, no. 7553, pp. 467-475, 2015.

[15] D. Bhattacharya, L. K. Cheng, and W. Xu, "Sparse machine learning discovery of dynamic differential equation of an esophageal swallowing robot," IEEE Transactions on Industrial Electronics, vol. 67, DOI 10.1109/TIE.2019.2928239, no. 6, pp. 4711-4720, 2019.

[16] S. L. Brunton, J. L. Proctor, and J. N. Kutz, "Discovering governing equations from data by sparse identification of nonlinear dynamical systems," Proceedings of the National Academy of Sciences, vol. 113, DOI 10.1073/pnas.1517384113, no. 15, pp. 3932-3937, 2016.

[17] S. L. Brunton, J. L. Proctor, and J. N. Kutz, "Sparse identification of nonlinear dynamics with control (SINDYc)," IFAC-PapersOnLine, vol. 49, DOI 10.1016/j.ifacol.2016.10.249, no. 18, pp. 710-715, 2016.

[18] E. Kaiser, J. N. Kutz, and S. L. Brunton, "Sparse identification of nonlinear dynamics for model predictive control in the low-data limit," Proceedings of the Royal Society A, vol. 474, DOI 10.1098/rspa.2018.0335, no. 2219, p. 20180335, 2018.

[19] S. Dirven, W. Xu, and L. K. Cheng, "Sinusoidal peristaltic waves in soft actuator for mimicry of esophageal swallowing," IEEE/ASME Transactions on Mechatronics, vol. 20, DOI 10.1109/TMECH.2014.2337291, no. 3, pp. 1331-1337, 2015.

[20] B. W. Brunton, L. A. Johnson, J. G. Ojemann, and J. N. Kutz, "Extracting spatial-temporal coherent patterns in large-scale neural recordings using dynamic mode decomposition," Journal of Neuroscience Methods, vol. 258, DOI 10.1016/j.jneumeth.2015.10.010, pp. 1 - 15, 2016.
[21] J. Misra and S. Pandey, "A mathematical model for oesophageal swallowing of a food-bolus," Mathematical and Computer Modelling, vol. 33, DOI 10.1016/S0895-7177(00)00295-8Get, no. 8-9, pp. 9971009, 2001.

[22] D. Takagi and N. Balmforth, "Peristaltic pumping of viscous fluid in an elastic tube," Journal of Fluid Mechanics, vol. 672, DOI 10.1017/S0022112010005914, p. 196, 2011.

[23] B. de Silva, K. Champion, M. Quade, J.-C. Loiseau, J. Kutz, and S. Brunton, "PySINDy: A python package for the sparse identification of nonlinear dynamical systems from data," Journal of Open Source Software, vol. 5, DOI 10.21105/joss.02104, no. 49, p. 2104, 2020.

[24] M. O'Leary, B. Hanson, and C. Smith, "Viscosity and non-newtonian features of thickened fluids used for dysphagia therapy," Journal of Food Science, vol. 75, DOI 0.1111/j.1750-3841.2010.01673.x, no. 6, pp. E330-E338, 2010.

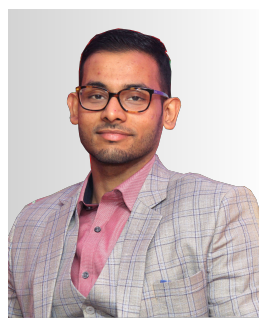

Dipankar Bhattacharya Dipankar Bhattacharya received the $B$.Tech. degree in electronics and communication engineering from the NERIST Itanagar, Arunachal Pradesh, India, in 2010, and the M.Tech. degree in electrical engineering from the IIT Roorkee, Roorkee, India, in 2013. $\mathrm{He}$ is currently working towards his Ph.D. thesis in mechatronics engineering with the University of Auckland, Auckland, New Zealand. His research interests include biologically inspired soft robots and machine learning.

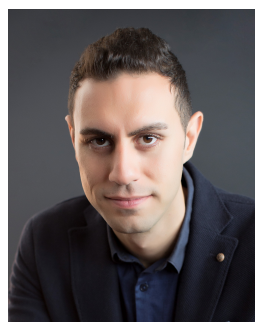

Ryman Hashem received the B.E. degree in mechatronics engineering from AMA International University, Salmabad, Bahrain, in 2012, and M.E. degree (First Class Hons.) in mechatronics engineering from the University of Auckland, Auckland, New Zealand, in 2015, where he is currently working toward the Ph.D. degree in mechatronics engineering. His current research interests include biologically-inspired soft robots design and control.

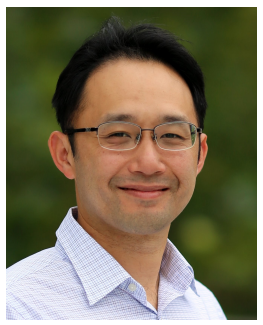

Leo K. Cheng received the B.E. (Hons.) degree in engineering science and the Ph.D. degree in bioengineering from the University of Auckland, Auckland, New Zealand, in 1997 and 2002, respectively. He is currently a Professor with Auckland Bioengineering Institute, Auckland. His main research interests include the understanding of electrophysiological events in the gastrointestinal tract and the heart, and their relationship to mechanical function.

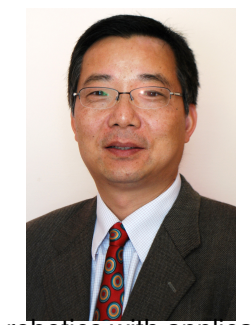

Weiliang $\mathrm{Xu}$ received the B.E. degree in manufacturing engineering and the M.E. degree in mechanical engineering from Southeast University, Nanjing, China, in 1982 and 1985, respectively, and the Ph.D. degree in mechatronics and robotics from Beihang University, Beijing, China, in 1988. He joined the University of Auckland, Auckland, New Zealand, in 2011, as the Chair in Mechatronics Engineering. His current research interests are mainly advanced mechatronics and robotics with applications in medicine and foods. 